\title{
Lie Algebras of Formal Power Series
}

\author{
Min Ho LEE \\ Department of Mathematics \\ University of Northern lowa \\ Cedar Falls, IA 50614 - USA \\ lee@math.uni.edu
}

Received: January 2, 2007

Accepted: April 26, 2007

\begin{abstract}
Pseudodifferential operators are formal Laurent series in the formal inverse $\partial^{-1}$ of the derivative operator $\partial$ whose coefficients are holomorphic functions. Given a pseudodifferential operator, the corresponding formal power series can be obtained by using some constant multiples of its coefficients. The space of pseudodifferential operators is a noncommutative algebra over $\mathbb{C}$ and therefore has a natural structure of a Lie algebra. We determine the corresponding Lie algebra structure on the space of formal power series and study some of its properties. We also discuss these results in connection with automorphic pseudodifferential operators, Jacobi-like forms, and modular forms for a discrete subgroup of $\mathrm{SL}(2, \mathbb{R})$.
\end{abstract}

Key words: Lie algebras, pseudodifferential operators, Jacobi-like forms, modular forms. 2000 Mathematics Subject Classification: 17B60, 11F50, 11F11, 35S05.

\section{Introduction}

Integrable nonlinear partial differential equations, or soliton equations, have been investigated in numerous papers during the past few decades, and they include many well-known equations in mathematical physics such as the Korteweg-de Vries (KdV) equation, Kadomtsev-Petviashvili (KP) equation, and nonlinear Schrödinger equation (see, e.g., $[2,3,5,8,10]$ ). One of the essential tools for studying soliton equations is the use of pseudodifferential operators, which are formal Laurent series, with holomorphic functions as coefficients, in the formal inverse $\partial^{-1}$ of the derivative operator

This research was supported in part by a UNI Summer Fellowship 
$\partial=d / d z$ with respect to the complex variable $z$ (see $[1,7,13,14])$. The space of pseudodifferential operators is in fact a noncommutative complex algebra in which the multiplication operation is given by the Leibniz rule. Thus the same space has the structure of an infinite-dimensional Lie algebra over $\mathbb{C}$ whose Lie bracket is the usual bracket operation associated to given noncommutative product.

Pseudodifferential operators containing only the terms of negative powers of $\partial$ may be referred to as integral pseudodifferential operators, since the inverse $\partial^{-1}$ of the derivative operator $\partial$ may be regarded as the antiderivative operator. The Lie algebra of integral pseudodifferential operators was studied by Khesin and Zakharevich [9] in connection with Poisson-Lie groups and Lie bialgebras. Indeed, they considered the Poisson-Lie structure obtained by extending this Lie algebra by the formal series $\log \partial$ and investigated relations between such a structure and Hamiltonians of KP and $\mathrm{KdV}$ flows. Our interest in integral pseudodifferential operators comes from the fact that they naturally correspond to formal power series. Indeed, a formal power series $\Phi(z, X)$ can be associated to an integral pseudodifferential operator $\Psi(z)$ in such a way that the coefficients of $\Phi(z, X)$ are some constant multiples of those of $\Psi(z)$. In this paper we are concerned with the Lie algebra structure of the space of formal power series corresponding to the same structure on the space of integral pseudodifferential operators described above.

Let $\mathcal{F}$ be the ring of holomorphic functions on $\mathbb{C}$. Given a positive integer $\delta$, let $\Psi \mathcal{D}(\mathcal{F})_{-\delta}$ be the space of integral pseudodifferential operators over $\mathcal{F}$ containing only the terms $\partial^{-k}$ for $k \geq \delta$. Similarly, let $\mathcal{F}[[X]]_{\delta}$ denote the space of formal power series in $X$ over $\mathcal{F}$ involving only the terms $X^{k}$ for $k \geq \delta$. If $\Phi(z, X)=\sum_{k=\delta}^{\infty} \phi_{k}(z) X^{k}$ is a formal power series belonging to $\mathcal{F}[[X]]_{\delta}$, we consider the corresponding integral pseudodifferential operator $\Psi(z)=\sum_{k=\delta}^{\infty} \psi_{k}(z) \partial^{-k}$, where $\phi_{k}=c_{k} \psi_{k} \in \mathcal{F}$ with $c_{k} \in \mathbb{C}$ for each $k \geq \delta$. Such a correspondence with $c_{k}=(-1)^{k} k !(k-1)$ ! was considered by Cohen, Manin, and Zagier [4], who showed, among other things, that it induces a correspondence between Jacobi-like forms and automorphic pseudodifferential operators. Thus there is a $\mathbb{C}$-linear isomorphism between $\Psi \mathcal{D}(\mathcal{F})_{-\delta}$ and $\mathcal{F}[[X]]_{\delta}$. In addition to being complex vector spaces, both $\Psi \mathcal{D}(\mathcal{F})_{-\delta}$ and $\mathcal{F}[[X]]_{\delta}$ are in fact algebras. The multiplication in $\Psi \mathcal{D}(\mathcal{F})_{-\delta}$ determined by the Leibniz rule is not commutative, while the usual multiplication on $\mathcal{F}[[X]]_{\delta}$ is commutative. The noncommutative product on $\Psi \mathcal{D}(\mathcal{F})_{-\delta}$ determines the usual Lie bracket under which $\Psi \mathcal{D}(\mathcal{F})_{-\delta}$ becomes a Lie algebra. One of the important properties of pseudodifferential operators is that there is a natural action of the group $\operatorname{SL}(2, \mathbb{C})$ on $\Psi \mathcal{D}(\mathcal{F})_{-\delta}$ induced by Möbius transformations of the complex plane $\mathbb{C}$. The isomorphism between $\Psi \mathcal{D}(\mathcal{F})_{-\delta}$ and $\mathcal{F}[[X]]_{\delta}$ can then be used to obtain the corresponding action of $\mathrm{SL}(2, \mathbb{C})$ on $\mathcal{F}[[X]]_{\delta}$, so that it becomes an isomorphism of $\mathrm{SL}(2, \mathbb{C})$-modules. From a number-theoretic point of view, an interesting feature of this action on formal power series is that it resembles the operation associated to one of the two transformation formulas for Jacobi forms in number theory (cf. [6]). It would be natural to consider various algebraic structures of $\mathcal{F}[[X]]_{\delta}$ corresponding to those of $\Psi \mathcal{D}(\mathcal{F})_{-\delta}$, and our focus in this paper is the Lie 
algebra structure, which was suggested by Cohen, Manin, and Zagier [4].

In order to study connections of pseudodifferential operators and formal power series with number theory, more specifically with modular forms, we need to consider those objects over the ring $R$ of holomorphic functions on the Poincare upper half plane $\mathcal{H}$. Thus in this case there are actions of $\operatorname{SL}(2, \mathbb{R})$ on $\Psi \mathcal{D}(R)_{\delta}$ and $R[[X]]_{\delta}$ which are compatible under an isomorphism between those two spaces. Let $\Gamma$ be a discrete subgroup of $\mathrm{SL}(2, \mathbb{R})$. Then $\Gamma$-invariant elements in $\Psi \mathcal{D}(R)_{\delta}$ and $R[[X]]_{\delta}$ are known as automorphic pseudodifferential operators and Jacobi-like forms, respectively, and they are closely linked to modular forms (see $[4,15]$ ). Indeed, given a Jacobi-like form $\Phi$ for $\Gamma$, a certain linear combination of derivatives of coefficients of $\Phi$ determines a modular form for $\Gamma$, and conversely, each coefficient of $\Phi$ can be expressed as a linear combination of derivatives of modular forms for $\Gamma$ of various weights. These relations can be used to establish a one-to-one correspondence between Jacobi-like forms and certain sequences of modular forms. Similarly, such sequences of modular forms also correspond to automorphic pseudodifferential operators. One of the applications of this correspondence is the construction of a lifting map from modular forms to Jacobilike forms or to automorphic pseudodifferential operators.

In this paper we derive the formula for the Lie bracket that determines the Lie algebra structure on $\mathcal{F}[[X]]_{\delta}$ described above and construct the Lie algebra homomorphism from $\mathfrak{s l}(2, \mathbb{C})$ to $\mathcal{F}[[X]]_{\delta}$ corresponding to the above-mentioned action of $\mathrm{SL}(2, \mathbb{C})$. We also determine equivariant maps from $\mathcal{F}$ to $\Psi \mathcal{D}(\mathcal{F})_{-\delta}$ and $\mathcal{F}[[X]]_{\delta}$ and discuss applications to automorphic pseudodifferential operators and Jacobi-like forms.

\section{Power series and pseudodifferential operators}

In this section we describe a correspondence between pseudodifferential operators and formal power series. We also discuss actions of $\operatorname{SL}(2, \mathbb{C})$ that are compatible under such a correspondence.

The group $\operatorname{SL}(2, \mathbb{C})$ acts on the complex plane $\mathbb{C}$ as usual by Möbius transformations, so that we have

$$
\gamma z=\frac{a z+b}{c z+d}
$$

for all $z \in \mathcal{H}$ and $\gamma=\left(\begin{array}{ll}a & b \\ c & d\end{array}\right) \in \operatorname{SL}(2, \mathbb{R})$. For the same $z$ and $\gamma$, we set

$$
\mathfrak{J}(\gamma, z)=c z+d, \quad \mathfrak{K}(\gamma, z)=c \mathfrak{J}(\gamma, z)^{-1}=\frac{c}{c z+d} .
$$

Then the resulting maps $\mathfrak{J}, \mathfrak{K}: \mathrm{SL}(2, \mathbb{C}) \times \mathbb{C}$ satisfy

$$
\mathfrak{J}\left(\gamma \gamma^{\prime}, z\right)=\mathfrak{J}\left(\gamma, \gamma^{\prime} z\right) \mathfrak{J}\left(\gamma^{\prime}, z\right), \quad \mathfrak{K}\left(\gamma \gamma^{\prime}, z\right)=\mathfrak{J}\left(\gamma^{\prime}, z\right)^{-2} \mathfrak{K}\left(\gamma, \gamma^{\prime} z\right)+\mathfrak{K}\left(\gamma^{\prime}, z\right)
$$

for all $\gamma, \gamma^{\prime} \in \mathrm{SL}(2, \mathbb{C})$ and $z \in \mathcal{H}$. 
Let $\mathcal{F}$ denote the ring of holomorphic functions on $\mathbb{C}$, and let $\mathcal{F}[[X]]$ be the complex algebra of formal power series in $X$ with coefficients in $\mathcal{F}$. Given a formal power series $\Phi(z, X) \in \mathcal{F}[[X]]$, an integer $\lambda$, and an element $\gamma \in \mathrm{SL}(2, \mathbb{C})$, we set

$$
\left(\left.\Phi\right|_{\lambda} ^{J} \gamma\right)(z, X)=\mathfrak{J}(\gamma, z)^{-\lambda} e^{-\mathfrak{K}(\gamma, z) X} \Phi\left(\gamma z, \mathfrak{J}(\gamma, z)^{-2} X\right)
$$

for all $z \in \mathcal{H}$. If $\gamma^{\prime}$ is another element of $\operatorname{SL}(2, \mathbb{C})$, then from $(2)$ we see that

$$
\left.\Phi\right|_{\lambda} ^{J}\left(\gamma \gamma^{\prime}\right)=\left.\left(\left.\Phi\right|_{\lambda} ^{J} \gamma\right)\right|_{\lambda} ^{J} \gamma^{\prime}
$$

hence the operation $\left.\right|_{\lambda} ^{J}$ determines a right action of $\operatorname{SL}(2, \mathbb{C})$ on $\mathcal{F}[[X]]$. If $\delta$ is a nonnegative integer, we set

$$
\mathcal{F}[[X]]_{\delta}=X^{\delta} \mathcal{F}[[X]],
$$

so that an element $\Phi(z, X) \in \mathcal{F}[[X]]_{\delta}$ can be written in the form

$$
\Phi(z, X)=\sum_{k=0}^{\infty} \phi_{k}(z) X^{k+\delta}
$$

with $\phi_{k} \in \mathcal{F}$ for each $k \geq 0$.

A pseudodifferential operator over $\mathcal{F}$ is a formal Laurent series in the formal inverse $\partial^{-1}$ of $\partial=d / d z$ with coefficients in $\mathcal{F}$ of the form

$$
\Psi(z)=\sum_{k=-\infty}^{u} h_{k}(z) \partial^{k}
$$

with $u \in \mathbb{Z}$ and $h_{k} \in \mathcal{F}$ for each $k \geq 0$. We denote by $\Psi \mathcal{D}(\mathcal{F})$ the space of all pseudodifferential operators over $\mathcal{F}$. Using the relation

$$
\frac{d}{d(\gamma z)}=\left(\frac{d(\gamma z)}{d z}\right)^{-1} \frac{d}{d z}=\mathfrak{J}(\gamma, z)^{2} \partial,
$$

we see that the group $\operatorname{SL}(2, \mathbb{C})$ acts on $\Psi \mathcal{D}(\mathcal{F})$ on the right by

$$
\Psi(z) \circ \gamma=\Psi(\gamma z)=\sum_{k=-\infty}^{u} h_{k}(\gamma z)\left(\mathfrak{J}(\gamma, z)^{2} \partial\right)^{k}
$$

for all $\gamma \in \mathrm{SL}(2, \mathbb{C})$, where $\mathfrak{J}(\gamma, z)$ is as in (1). If $\alpha$ is an integer, we denote by $\Psi \mathcal{D}(\mathcal{F})_{\alpha}$ the subspace of $\Psi \mathcal{D}(\mathcal{F})$ consisting of the pseudodifferential operators of the form

$$
\sum_{k=0}^{\infty} \psi_{k}(z) \partial^{\alpha-k}
$$

with $\psi_{k} \in \mathcal{F}$ for all $k \geq 0$. 
We now introduce an isomorphism between the space of formal power series and that of pseudodifferential operators defined as follows, which was considered by Cohen, Manin, and Zagier in [4]. Given a formal power series

$$
F(z, X)=\sum_{k=0}^{\infty} f_{k}(z) X^{k+\delta} \in \mathcal{F}[[X]]_{\delta}
$$

and a pseudodifferential operator

$$
\Psi(z)=\sum_{k=0}^{\infty} \psi_{k}(z) \partial^{-k-\varepsilon} \in \Psi \mathcal{D}(\mathcal{F})_{-\varepsilon}
$$

with $\delta, \varepsilon>0$, we set

$$
\begin{aligned}
& \left(\mathcal{L}_{\xi}^{\partial} F\right)(z)=\sum_{k=0}^{\infty} C_{k+\delta+\xi} f_{k}(z) \partial^{-k-\delta-\xi} \\
& \left(\mathcal{L}_{\xi}^{X} \Psi\right)(z, X)=\sum_{k=0}^{\infty} C_{k+\varepsilon}^{-1} \psi_{k}(z) X^{k+\varepsilon-\xi}
\end{aligned}
$$

for each nonnegative integer $\xi$, where $C_{\eta}$ with $\eta>0$ denotes the integer

$$
C_{\eta}=(-1)^{\eta} \eta !(\eta-1) !
$$

Then it can be easily seen that

$$
\left(\mathcal{L}_{\xi}^{X} \circ \mathcal{L}_{\xi}^{\partial}\right) F=F, \quad\left(\mathcal{L}_{\xi}^{\partial} \circ \mathcal{L}_{\xi}^{X}\right) \Psi=\Psi,
$$

and therefore the resulting maps

$$
\mathcal{L}_{\xi}^{\partial}: \mathcal{F}[[X]]_{\delta} \rightarrow \Psi \mathcal{D}(\mathcal{F})_{-\delta-\xi}, \quad \mathcal{L}_{\xi}^{X}: \Psi \mathcal{D}(\mathcal{F})_{-\varepsilon} \rightarrow \mathcal{F}[[X]]_{\varepsilon+\xi}
$$

are linear isomorphisms.

Proposition 1.1. Let $F(z, X) \in \mathcal{F}[[X]]_{\delta}$ and $\Psi(z) \in \Psi \mathcal{D}(\mathcal{F})_{-\varepsilon}$ with $\delta, \varepsilon>0$. If $\xi$ is a nonnegative integer, we have

$$
\left(\mathcal{L}_{\xi}^{\partial} F\right) \circ \gamma=\mathcal{L}_{\xi}^{\partial}\left(\left.F\right|_{2 \xi} ^{J} \gamma\right),\left.\quad\left(\mathcal{L}_{\xi}^{X} \Psi\right)\right|_{2 \xi} ^{J} \gamma=\mathcal{L}_{\xi}^{X}(\Psi \circ \gamma)
$$

for all $\gamma \in \mathrm{SL}(2, \mathbb{C})$.

Proof. Given $\gamma \in \mathrm{SL}(2, \mathbb{C}), z \in \mathcal{H}$, and $F(z, X) \in \mathcal{F}[[X]]_{\delta}$ as in (5), using (4), we have

$$
\left(\left(\mathcal{L}_{\xi}^{\partial} F\right) \circ \gamma\right)(z)=\left(\mathcal{L}_{\xi}^{\partial} F\right)\left(\gamma z, \mathfrak{J}(\gamma, z)^{2} \partial\right)=\sum_{k=0}^{\infty} C_{k+\delta+\xi} f_{k}(\gamma z)\left(\mathfrak{J}(\gamma, z)^{2} \partial\right)^{-k-\delta-\xi} .
$$


If $m$ is an integer, it can be shown by induction that

$$
\left(\mathfrak{J}(\gamma, z)^{2} \partial\right)^{m}=\sum_{r=0}^{\infty} r !\left(\begin{array}{c}
m \\
r
\end{array}\right)\left(\begin{array}{c}
m-1 \\
r
\end{array}\right) \mathfrak{J}(\gamma, z)^{2 m} \mathfrak{K}(\gamma, z)^{r} \partial^{m-r} .
$$

Using this, we obtain

$$
\begin{aligned}
\left(\left(\mathcal{L}_{\xi}^{\partial} F\right) \circ \gamma\right)(z)= & \sum_{k=0}^{\infty} \sum_{r=0}^{\infty} C_{k+\delta+\xi} f_{k}(\gamma z) r !\left(\begin{array}{c}
-k-\delta-\xi \\
r
\end{array}\right)\left(\begin{array}{c}
-k-\delta-\xi-1 \\
r
\end{array}\right) \\
& \times \mathfrak{J}(\gamma, z)^{-2 k-2 \delta-2 \xi} \mathfrak{K}(\gamma, z)^{r} \partial^{-k-\delta-\xi-r} \\
= & \sum_{k=0}^{\infty} \sum_{r=0}^{k} C_{k-r+\delta+\xi} f_{k-r}(\gamma z) r !\left(\begin{array}{c}
r-k-\delta-\xi \\
r
\end{array}\right)\left(\begin{array}{c}
r-k-\delta-\xi-1 \\
r
\end{array}\right) \\
& \times \mathfrak{J}(\gamma, z)^{-2 k-2 \delta-2 \xi+2 r} \mathfrak{K}(\gamma, z)^{r} \partial^{-k-\delta-\xi} .
\end{aligned}
$$

From this and the identity

$$
\left(\begin{array}{c}
-\alpha \\
r
\end{array}\right)=\frac{(-1)^{r}(\alpha-1) !}{r !(\alpha-1-r) !}
$$

for $\alpha \geq 1$, we see that

$$
\begin{aligned}
\left(\left(\mathcal{L}_{\xi}^{\partial} F\right)\right. & \circ \gamma)(z) \\
& =\sum_{k=0}^{\infty} \sum_{r=0}^{k} \frac{(-1)^{r}}{r !} C_{k+\delta+\xi} \mathfrak{J}(\gamma, z)^{-2 k-2 \delta-2 \xi+2 r} \mathfrak{K}(\gamma, z)^{r} f_{k-r}(\gamma z) \partial^{-k-\delta-\xi} .
\end{aligned}
$$

On the other hand, using (3), we have

$$
\begin{aligned}
\left(\left.F\right|_{2 \xi} ^{J} \gamma\right)(z, X) & =\mathfrak{J}(\gamma, z)^{-2 \xi}\left(\sum_{r=0}^{\infty} \frac{(-1)^{r}}{r !} \mathfrak{K}(\gamma, z)^{r} X^{r}\right) \sum_{k=0}^{\infty} f_{k}(\gamma z)\left(\mathfrak{J}(\gamma, z)^{-2} X\right)^{k+\delta} \\
& =\sum_{k=0}^{\infty} \sum_{r=0}^{\infty} \frac{(-1)^{r}}{r !} \mathfrak{J}(\gamma, z)^{-2 k-2 \delta-2 \xi} \mathfrak{K}(\gamma, z)^{r} f_{k}(\gamma z) X^{k+r+\delta} \\
& =\sum_{k=0}^{\infty} \sum_{r=0}^{k} \frac{(-1)^{r}}{r !} \mathfrak{J}(\gamma, z)^{-2 k-2 \delta-2 \xi+2 r} \mathfrak{K}(\gamma, z)^{r} f_{k-r}(\gamma z) X^{k+\delta} .
\end{aligned}
$$

Applying $\mathcal{L}_{\xi}^{\partial}$ to this relation and comparing it with (10), we see that

$$
\mathcal{L}_{\xi}^{\partial}\left(\left.F\right|_{2 \xi} ^{J} \gamma\right)(z)=\left(\left(\mathcal{L}_{\xi}^{\partial} F\right) \circ \gamma\right)(z)
$$

which verifies the first relation in (9). We now apply this to $F(z, X)=\left(\mathcal{L}_{\xi}^{X} \Psi\right)(z, X)$, so that

$$
\mathcal{L}_{\xi}^{\partial}\left(\left.\left(\mathcal{L}_{\xi}^{X} \Psi\right)\right|_{2 \xi} ^{J} \gamma\right)=\mathcal{L}_{\xi}^{\partial}\left(\mathcal{L}_{\xi}^{X} \Psi\right) \circ \gamma=\Psi \circ \gamma
$$

Then the second relation in (9) is obtained by applying $\mathcal{L}_{\xi}^{X}$ to this relation. 


\section{Lie algebras of power series}

In this section we introduce a Lie algebra structure on the space of power series that is compatible with the natural Lie algebra structure on the noncommutative algebra of pseudodifferential operators. We determine an explicit formula for the associated Lie bracket.

Given pseudodifferential operators $\Psi(z), \Phi(z) \in \Psi \mathcal{D}(\mathcal{F})_{-\delta}$ with $\delta>0$, we set

$$
[\Psi(z), \Phi(z)]^{\partial}=\Psi(z) \Phi(z)-\Phi(z) \Psi(z),
$$

which belongs to $\Psi \mathcal{D}(\mathcal{F})_{-2 \delta}$. Then this formula determines a skew-symmetric bilinear map

$$
[,]^{\partial}: \Psi \mathcal{D}(\mathcal{F})_{-\delta} \times \Psi \mathcal{D}(\mathcal{F})_{-\delta} \rightarrow \Psi \mathcal{D}(\mathcal{F})_{-2 \delta}
$$

which provides $\Psi \mathcal{D}(\mathcal{F})$ with a structure of a complex Lie algebra.

Assuming that

$$
\Psi(z)=\sum_{k=0}^{\infty} \psi_{k}(z) \partial^{-k-\delta}, \quad \Phi(z)=\sum_{k=0}^{\infty} \phi_{k}(z) \partial^{-k-\delta}
$$

we have

$$
\Psi(z) \Phi(z)=\sum_{k=0}^{\infty} \sum_{\ell=0}^{\infty} \sum_{q=0}^{\infty}\left(\begin{array}{c}
-k-\delta \\
q
\end{array}\right) \psi_{k}(z) \phi_{\ell}^{(q)}(z) \partial^{-k-\ell-q-2 \delta} .
$$

Changing the indices from $k, \ell, q$ to $r, p, q$ with $r=k+\ell+q$ and $p=\ell+q$, we obtain

$$
\Psi(z) \Phi(z)=\sum_{r=0}^{\infty} \sum_{p=0}^{r} \sum_{q=0}^{p}\left(\begin{array}{c}
-r-\delta+p \\
q
\end{array}\right) \psi_{r-p}(z) \phi_{p-q}^{(q)}(z) \partial^{-r-2 \delta} .
$$

Hence we see that

$$
[\Psi(z), \Phi(z)]^{\partial}=\Psi(z) \Phi(z)-\Phi(z) \Psi(z)=\sum_{r=0}^{\infty} \eta(\Psi, \Phi)_{r} \partial^{-r-2 \delta},
$$

where

$$
\eta(\Psi, \Phi)_{r}=\sum_{p=0}^{r} \sum_{q=0}^{p}\left(\begin{array}{c}
-r-\delta+p \\
q
\end{array}\right)\left(\psi_{r-p}(z) \phi_{p-q}^{(q)}(z)-\phi_{r-p}(z) \psi_{p-q}^{(q)}(z)\right)
$$

for all $r \geq 0$.

We now consider formal power series $F(z, X)$ and $G(z, X)$ belonging to $\mathcal{F}[[X]]_{\delta}$ given by

$$
F(z, X)=\sum_{k=0}^{\infty} f_{k}(z) X^{k+\delta}, \quad G(z, X)=\sum_{k=0}^{\infty} g_{k}(z) X^{k+\delta}
$$


We then define the formal power series $[F(z, X), G(z, X)]^{X}$ by

$$
\begin{aligned}
{[F(z, X), G(z, X)]^{X}=\sum_{r=0}^{\infty} \sum_{p=0}^{r} } & \sum_{q=0}^{p}\left(\begin{array}{c}
-r-\delta-\xi+p \\
q
\end{array}\right) \frac{C_{r-p+\delta+\xi} C_{p-q+\delta+\xi}}{C_{r+2 \delta+2 \xi}} \\
& \times\left(f_{r-p}(z) g_{p-q}^{(q)}(z)-g_{r-p}(z) f_{p-q}^{(q)}(z)\right) X^{r+2 \delta}
\end{aligned}
$$

Theorem 2.1. The formula (13) determines a bilinear map

$$
[,]^{X}: \mathcal{F}[[X]]_{\delta} \times \mathcal{F}[[X]]_{\delta} \rightarrow \mathcal{F}[[X]]_{2 \delta}
$$

of formal power series satisfying

$$
\mathcal{L}_{2 \xi}^{\partial}\left([F(z, X), G(z, X)]^{X}\right)=\left[\mathcal{L}_{\xi}^{\partial}(F(z, X)), \mathcal{L}_{\xi}^{\partial}(G(z, X))\right]^{\partial}
$$

for all $F(z, X), G(z, X) \in \mathcal{F}[[X]]_{\delta}$ and $\xi \geq 0$.

Proof. Let $F(z, X), G(z, X) \in \mathcal{F}[[X]]_{\delta}$ be given by (12). By (6) the pseudodifferential operators $\mathcal{L}_{\xi}^{\partial}(F(z, X))$ and $\mathcal{L}_{\xi}^{\partial}(G(z, X))$ are given by

$\mathcal{L}_{\xi}^{\partial}(F(z, X))=\sum_{k=0}^{\infty} C_{k+\delta+\xi} f_{k}(z) \partial^{-k-\delta-\xi}, \quad \mathcal{L}_{\xi}^{\partial}(G(z, X))=\sum_{k=0}^{\infty} C_{k+\delta+\xi} g_{k}(z) \partial^{-k-\delta-\xi}$

for each $\xi \geq 0$. Thus, from these relations and (11) we obtain

$$
\begin{aligned}
& {\left[\mathcal{L}_{\xi}^{\partial}(F(z, X)), \mathcal{L}_{\xi}^{\partial}(G(z, X))\right]^{\partial} } \\
&=\sum_{r=0}^{\infty} \sum_{p=0}^{r} \sum_{q=0}^{p}\left(\begin{array}{c}
- \\
q-\delta-\xi+p \\
q
\end{array}\right) C_{r-p+\delta+\xi} C_{p-q+\delta+\xi} \\
& \quad \times\left(f_{r-p}(z) g_{p-q}^{(q)}(z)-g_{r-p}(z) f_{p-q}^{(q)}(z)\right) \partial^{-r-2 \delta-2 \xi}
\end{aligned}
$$

From this, (7) and (13) it follows that

$$
[F(z, X), G(z, X)]^{X}=\mathcal{L}_{2 \xi}^{X}\left[\mathcal{L}_{\xi}^{\partial}(F(z, X)), \mathcal{L}_{\xi}^{\partial}(G(z, X))\right]^{\partial}
$$

In particular, we see that $[F(z, X), G(z, X)]^{X} \in \mathcal{F}[[X]]_{2 \delta}$ and that (14) holds.

From Theorem 2.1 it follows that $[,]^{X}$ is a Lie bracket on $\mathcal{F}[[X]]$, and therefore $\mathcal{F}[[X]]$ together with this bracket is a complex Lie algebra. 


\section{Lie algebra homomorphisms}

Let $\mathcal{F}$ be the space of holomorphic functions on the complex plane $\mathbb{C}$, and let $\Psi \mathcal{D}(\mathcal{F})$ and $\mathcal{F}[[X]]$ be as in section 1 . In this section we determine a Lie algebra homomorphism from $\mathfrak{s l}(2, \mathbb{C})$ to $\mathcal{F}[[X]]$ which corresponds to the natural action of $\mathrm{SL}(2, \mathbb{C})$ on $\Psi \mathcal{D}(\mathcal{F})$.

Let $\operatorname{End}(\Psi \mathcal{D}(\mathcal{F}))$ be the space of complex linear endomorphisms of $\Psi \mathcal{D}(\mathcal{F})$, and let $\operatorname{Aut}(\Psi \mathcal{D}(\mathcal{F}))$ be the group of invertible elements in $\operatorname{End}(\Psi \mathcal{D}(\mathcal{F}))$. We consider the left action $\rho: \operatorname{SL}(2, \mathbb{C}) \rightarrow \operatorname{Aut}(\Psi \mathcal{D}(\mathcal{F}))$ of $\operatorname{SL}(2, \mathbb{C})$ on $\Psi \mathcal{D}(\mathcal{F})$ defined by

$$
(\rho(\gamma) \Psi)(z)=\Psi(z) \circ \gamma^{-1}=\Psi\left(\gamma^{-1} z\right)
$$

for all $\gamma \in \operatorname{SL}(2, \mathbb{C})$ and $\Psi(z) \in \Psi \mathcal{D}(\mathcal{F})$, where $\Psi(z) \circ \gamma^{-1}$ is as in (4). Then, as usual, the homomorphism $\rho$ determines a linear map

$$
\sigma: \mathfrak{s l}(2, \mathbb{C}) \rightarrow \operatorname{End}(\Psi \mathcal{D}(\mathcal{F}))
$$

defined by

$$
\sigma(v) \Psi(z)=\left.\frac{d}{d t} \rho\left(e^{t v}\right) \Psi(z)\right|_{t=0}=\left.\frac{d}{d t} \Psi\left(e^{-t v} z\right)\right|_{t=0}
$$

for $v \in \mathfrak{s l}(2, \mathbb{C}), \Psi \in \Psi \mathcal{D}(\mathcal{F})$ and $z \in \mathbb{C}$, which satisfies

$$
\sigma([v, w])=\sigma(v) \sigma(w)-\sigma(w) \sigma(v)
$$

for all $v, w \in \mathfrak{s l}(2, \mathbb{C})$. We shall show below that each endomorphism $\sigma(v) \in$ $\operatorname{End}(\Psi \mathcal{D}(\mathcal{F}))$ of $\Psi \mathcal{D}(\mathcal{F})$ is simply multiplication by an element of $\Psi \mathcal{D}(\mathcal{F})$ on the left.

We consider the standard basis $\left\{h, e^{+}, e^{-}\right\}$for the Lie algebra $\mathfrak{s l}(2, \mathbb{C})$ given by

$$
h=\left(\begin{array}{cc}
1 & 0 \\
0 & -1
\end{array}\right), \quad e^{+}=\left(\begin{array}{ll}
0 & 1 \\
0 & 0
\end{array}\right), \quad e^{-}=\left(\begin{array}{ll}
0 & 0 \\
1 & 0
\end{array}\right) .
$$

Then these vectors satisfy the relations

$$
\left[h, e^{+}\right]=2 e^{+}, \quad\left[h, e^{-}\right]=-2 e^{-}, \quad\left[e^{+}, e^{-}\right]=h .
$$

Lemma 3.1. Let $v \in \mathfrak{s l}(2, \mathbb{C})$ be an element given by

$$
v=K_{1} h+K_{2} e_{+}+K_{3} e^{-}
$$

for some $K_{1}, K_{2}, K_{3} \in \mathbb{C}$. Then we have

$$
\sigma(v)=\left(-2 K_{1} z-K_{2}+K_{3} z^{2}\right) \partial
$$

with $\partial=d / d z$. 
Proof. For the basis vectors in (16) we see that

$$
e^{-t h}=\left(\begin{array}{cc}
e^{-t} & 0 \\
0 & e^{t}
\end{array}\right), \quad e^{-t e^{+}}=\left(\begin{array}{cc}
1 & -t \\
0 & 1
\end{array}\right), \quad e^{-t e^{-}}=\left(\begin{array}{cc}
1 & 0 \\
-t & 1
\end{array}\right) .
$$

From these relations and (15) we obtain

$$
\begin{aligned}
\sigma(h) \Psi(z) & =\left.\frac{d}{d t} \Psi\left(e^{-2 t} z\right)\right|_{t=0}=-\left.2 e^{-2 t} z \partial \Psi\left(e^{-2 t} z\right)\right|_{t=0}=-2 z \partial \Psi(z), \\
\sigma\left(e^{+}\right) \Psi(z) & =\left.\frac{d}{d t} \Psi(z-t)\right|_{t=0}=-\left.\partial \Psi(z-t)\right|_{t=0}=-\partial \Psi(z) \\
\sigma\left(e^{-}\right) \Psi(z) & =\left.\frac{d}{d t} \Psi\left(\frac{z}{-t z+1}\right)\right|_{t=0}=\left.\frac{z^{2}}{(-t z+1)^{2}} \partial \Psi\left(\frac{z}{-t z+1}\right)\right|_{t=0}=z^{2} \partial \Psi(z)
\end{aligned}
$$

for all $z \in \mathcal{H}$. Thus we have

$$
\sigma(h)=-2 z \partial, \quad \sigma\left(e^{+}\right)=-\partial, \quad \sigma\left(e^{-}\right)=z^{2} \partial ;
$$

hence the lemma follows by linear extension.

Lemma 3.1 shows in particular that each $\sigma(v)$ with $v \in \mathfrak{s l}(2, \mathbb{C})$ may be regarded as an element of $\Psi \mathcal{D}(\mathcal{F})$ itself. On the other hand, the noncommutative complex algebra $\Psi \mathcal{D}(\mathcal{F})$ has the structure of a Lie algebra whose bracket operation is given by

$$
\left[\Psi_{1}, \Psi_{2}\right]^{\partial}=\Psi_{1} \Psi_{2}-\Psi_{2} \Psi_{1}
$$

for all $\Psi_{1}, \Psi_{2} \in \operatorname{End}(\Psi \mathcal{D}(\mathcal{F}))$. Thus we see that the map $\sigma$ may be regarded as the Lie algebra homomorphism

$$
\sigma: \mathfrak{s l}(2, \mathbb{C}) \rightarrow \Psi \mathcal{D}(\mathcal{F})
$$

Given $\xi \geq 0$, we now set

$$
\sigma_{X}=\mathcal{L}_{\xi}^{X} \circ \sigma: \mathfrak{s l}(2, \mathbb{C}) \rightarrow \mathcal{F}[[X]]
$$

where $\mathcal{L}_{\xi}^{X}$ is as in (7).

Lemma 3.2. The map $\sigma_{X}$ in (18) is a Lie algebra homomorphism, where the Lie algebra structure on $\mathcal{F}[[X]]$ is given by the bracket in (13).

Proof. If $u, v \in \mathfrak{s l}(2, \mathbb{C})$, using (14), we see that

$$
\begin{aligned}
{\left[\left(\sigma_{X}(v)\right)(z, X),\left(\sigma_{X}(w)\right)(z, X)\right]^{X} } & =\mathcal{L}_{2 \xi}^{X}\left[\left(\left(\mathcal{L}_{\xi}^{\partial} \circ \sigma_{X}\right)(v)\right)(z),\left(\left(\mathcal{L}_{\xi}^{\partial} \circ \sigma_{X}\right)(w)\right)(z)\right]^{\partial} \\
& =\mathcal{L}_{2 \xi}^{X}[(\sigma(v))(z),(\sigma(w))(z)]^{\partial} \\
& =\left(\mathcal{L}_{2 \xi}^{X} \circ \sigma\right)([v, w])(z)=\left(\sigma_{X}([v, w])\right)(z, X)
\end{aligned}
$$

hence the lemma follows. 
From Lemma 3.2 it follows that the Lie algebra $\mathfrak{s l}(2, \mathbb{C})$ acts on $\mathcal{F}[[X]]$ by the adjoint representation, so that

$$
v \cdot F(z, X)=\operatorname{ad}(v) F(z, X)=\left[\left(\sigma_{X} v\right)(z, X), F(z, X)\right]^{X}
$$

for $v \in \mathfrak{s l}(2, \mathbb{C})$ and $F(z, X) \in \mathcal{F}[[X]]$. The next proposition provides formulas for this representation for basis vectors.

Proposition 3.3. Given a formal power series $F(z, X)=\sum_{k=0}^{\infty} f_{k}(z) X^{k+\delta} \in$ $\mathcal{F}[[X]]_{\delta}$, we have

$$
\begin{aligned}
h \cdot F(z, X) & =-2 \sum_{k=0}^{\infty}\left(z\left(\partial f_{k}\right)(z)+(k+\delta+\xi) f_{k}(z)\right) X^{k+\delta}, \\
e^{+} \cdot F(z, X) & =-\sum_{k=0}^{\infty}\left(\partial f_{k}\right)(z) X^{k+\delta}, \\
e^{-} \cdot F(z, X) & =\sum_{k=0}^{\infty}\left(z^{2}\left(\partial f_{k}\right)(z)+2(k+\delta+\xi) f_{k}(z)-f_{k-1}(z)\right) X^{k+\delta}
\end{aligned}
$$

with $f_{-1}=0$, where $h, e^{+}, e^{-} \in \mathfrak{s l}(2, \mathbb{C})$ are the standard basis vectors in $(16)$.

Proof. Consider an element of the form $h(z) \partial^{\omega} \in \Psi \mathcal{D}(\mathcal{F})$ with $\omega \in \mathbb{Z}$. Then, using (17) and (19), we have

$$
\begin{aligned}
-\frac{1}{2} \operatorname{ad}(\sigma(h))\left(h(z) \partial^{\omega}\right)= & {\left[z \partial, h(z) \partial^{\omega}\right]=z \partial\left(h(z) \partial^{\omega}\right)-h(z) \partial^{\omega}(z \partial) } \\
= & z(\partial h)(z) \partial^{\omega}+z h(z) \partial^{\omega+1}-h(z) z \partial^{\omega+1}-\omega h(z) \partial^{\omega} \\
= & (z(\partial h)(z)-\omega h(z)) \partial^{\omega}, \\
-\operatorname{ad}\left(\sigma\left(e^{+}\right)\right)\left(h(z) \partial^{\omega}\right)= & {\left[\partial, h(z) \partial^{\omega}\right]=\partial\left(h(z) \partial^{\omega}\right)-h(z) \partial^{\omega+1} } \\
= & (\partial h)(z) \partial^{\omega}+h(z) \partial^{\omega+1}-h(z) \partial^{\omega+1}=(\partial h)(z) \partial^{\omega}, \\
\operatorname{ad}\left(\sigma\left(e^{-}\right)\right)\left(h(z) \partial^{\omega}\right)= & {\left[z^{2} \partial, h(z) \partial^{\omega}\right]=z^{2} \partial\left(h(z) \partial^{\omega}\right)-h(z) \partial^{\omega}\left(z^{2} \partial\right) } \\
= & z^{2}(\partial h(z)) \partial^{\omega}+z^{2} h(z) \partial^{\omega+1} \\
& -h(z)\left(z^{2} \partial^{\omega+1}+2 \omega z \partial^{\omega}+\omega(\omega-1) \partial^{\omega-1}\right) \\
= & \left(z^{2}(\partial h(z))-2 \omega z h(z)\right) \partial^{\omega}-\omega(\omega-1) h(z) \partial^{\omega-1} .
\end{aligned}
$$


If $\Psi(z)=\sum_{\nu=0}^{\infty} \psi_{\nu}(z) \partial^{\eta-\nu} \in \Psi \mathcal{D}(\mathcal{F})_{\eta}$ with $\eta>0$, from the above relations we obtain

$$
\begin{aligned}
\operatorname{ad}(\sigma(h))(\Psi(z)) & =-2 \sum_{\nu=0}^{\infty}\left(z\left(\partial \psi_{\nu}\right)(z)-(\eta-\nu) \psi_{\nu}(z)\right) \partial^{\eta-\nu} \\
\operatorname{ad}\left(\sigma\left(e^{+}\right)\right)(\Psi(z))= & -\sum_{\nu=0}^{\infty}\left(\partial \psi_{\nu}\right)(z) \partial^{\eta-\nu} \\
\operatorname{ad}\left(\sigma\left(e^{-}\right)\right)(\Psi(z))= & \sum_{\nu=0}^{\infty}\left(z^{2}\left(\partial \psi_{\nu}\right)(z)-2(\eta-\nu) z \psi_{\nu}(z)\right. \\
& \left.-(\eta-\nu)(\eta-\nu+1) \psi_{\nu-1}(z)\right) \partial^{\eta-\nu}
\end{aligned}
$$

with $\psi_{-1}=0$. We now consider a formal power series

$$
F(z, X)=\sum_{k=0}^{\infty} f_{k}(z) X^{k+\delta} \in \mathcal{F}[[X]]_{\delta}
$$

with $\delta>0$. Then, given $\xi \geq 0$, by using (6) we may write

$$
\mathcal{L}_{\xi}^{\partial}(F(z, X))=\sum_{k=0}^{\infty} C_{k+\delta+\xi} f_{k}(z) \partial^{-k-\delta-\xi},
$$

where $C_{k+\delta+\xi}$ is as in (8). Thus, using (23), (24), and (25), we have

$$
\begin{aligned}
-\frac{1}{2} \operatorname{ad}(\sigma(h))\left(\mathcal{L}_{\xi}^{\partial}(F(z, X))\right)=\sum_{k=0}^{\infty} C_{k+\delta+\xi}\left(z\left(\partial f_{k}\right)(z)+(k+\delta+\xi) f_{k}(z)\right) \partial^{-k-\delta-\xi}, \\
-\operatorname{ad}\left(\sigma\left(e^{+}\right)\right)\left(\mathcal{L}_{\xi}^{\partial}(F(z, X))\right)=\sum_{k=0}^{\infty} C_{k+\delta+\xi}\left(\partial f_{k}\right)(z) \partial^{-k-\delta-\xi} \\
\operatorname{ad}\left(\sigma\left(e^{-}\right)\right)\left(\mathcal{L}_{\xi}^{\partial}(F(z, X))\right) \\
=\sum_{k=0}^{\infty}\left(C_{k+\delta+\xi} z^{2}\left(\partial f_{k}\right)(z)+2 C_{k+\delta+\xi}(k+\delta+\xi) z f_{k}(z)\right. \\
\left.\quad-(k+\delta+\xi)(k+\delta+\xi-1) C_{k+\delta+\xi-1} f_{k-1}(z)\right) \partial^{-k-\delta-\xi} \\
=\sum_{k=0}^{\infty} C_{k+\delta+\xi}\left(z^{2}\left(\partial f_{k}\right)(z)+2(k+\delta+\xi) z f_{k}(z)+f_{k-1}(z)\right) \partial^{-k-\delta-\xi}
\end{aligned}
$$


with $f_{-1}=0$. From the above identities and (7) we see that

$$
\begin{aligned}
-\frac{1}{2}\left(\mathcal{L}_{\xi}^{X} \circ \operatorname{ad}(\sigma(h)) \circ \mathcal{L}_{\xi}^{\partial}\right)(F(z, X))=\sum_{k=0}^{\infty}\left(z\left(\partial f_{k}\right)(z)+(k+\delta+\xi) f_{k}(z)\right) X^{k+\delta} \\
-\left(\mathcal{L}_{\xi}^{X} \circ \operatorname{ad}\left(\sigma\left(e^{+}\right)\right) \circ \mathcal{L}_{\xi}^{\partial}\right)(F(z, X))=\sum_{k=0}^{\infty}\left(\partial f_{k}\right)(z) X^{k+\delta} \\
\left(\mathcal{L}_{\xi}^{X} \circ \operatorname{ad}\left(\sigma\left(e^{-}\right) \circ \mathcal{L}_{\xi}^{\partial}\right)(F(z, X))\right. \\
=\sum_{k=0}^{\infty}\left(z^{2}\left(\partial f_{k}\right)(z)+2(k+\delta+\xi) f_{k}(z)+f_{k-1}(z)\right) X^{k+\delta}
\end{aligned}
$$

Thus the lemma follows by comparing these identities with (20), (21), and (22).

\section{Equivariant splittings}

For each formal power series $F(z, X) \in \mathcal{F}[[X]]_{\delta}$ with $\delta>0$, by taking its coefficient of $X^{\delta}$, we obtain a surjective linear map from $\mathcal{F}[[X]]_{\delta}$ to $\mathcal{F}$. In this section we obtain a right inverse of this map that is equivariant with respect to $\mathrm{SL}(2, \mathbb{C})$.

If $\left\{h, e^{+}, e^{-}\right\}$is the standard basis in $(16)$ for $\mathfrak{s l}(2, \mathbb{C})$, using the inner product

$$
\langle v, w\rangle=\operatorname{tr}(v, w)
$$

for $v, w \in \mathfrak{s l}(2, \mathbb{C})$, we see that

$$
\langle h, h\rangle=2, \quad\left\langle h, e^{+}\right\rangle=0, \quad\left\langle h, e^{-}\right\rangle=0, \quad\left\langle e^{+}, e^{-}\right\rangle=1 .
$$

Thus the corresponding dual basis $\left\{h^{*},\left(e^{+}\right)^{*},\left(e^{-}\right)^{*}\right\}$ is given by

$$
h^{*}=h / 2, \quad\left(e^{+}\right)^{*}=e^{-}, \quad\left(e^{-}\right)^{*}=e^{+},
$$

and the associated Casimir operator $\mathcal{C}$ belonging to the universal enveloping algebra $U(\mathfrak{s l}(2, \mathbb{C}))$ of $\mathfrak{s l}(2, \mathbb{C})$ can be written as

$$
\mathcal{C}=h h^{*}+e^{+}\left(e^{+}\right)^{*}+e^{-}\left(e^{-}\right)^{*}=\frac{1}{2} h^{2}+e^{+} e^{-}+e^{-} e^{+} .
$$

The action of $\mathfrak{s l}(2, \mathbb{C})$ given by $(19)$ induces the action of $\mathcal{C}$ on $\mathcal{F}[[X]]_{\delta}$.

Proposition 4.1. If $F(z, X)=\sum_{k=0}^{\infty} f_{k}(z) X^{k+\delta} \in \mathcal{F}[[X]]_{\delta}$ with $\delta>0$, then we have

$$
\mathcal{C} \cdot F(z, X)=2 \sum_{k=0}^{\infty}\left((k+\delta+\xi)(k+\delta+\xi-1) f_{k}(z)-\left(\partial f_{k-1}\right)(z)\right) X^{k+\delta}
$$

with $f_{-1}=0$. 
Proof. Using (20), (21), and (22), we have

$$
\begin{aligned}
h^{2} \cdot F(z, X)= & 4 \sum_{k=0}^{\infty}\left(z \partial\left(z\left(\partial f_{k}\right)(z)+(k+\delta+\xi) f_{k}(z)\right)\right. \\
& +(k+\delta+\xi)\left(z\left(\partial f_{k}\right)(z)+(k+\delta+\xi) f_{k}(z)\right) X^{k+\delta} \\
= & 4 \sum_{k=0}^{\infty}\left(z\left(\left(\partial f_{k}\right)(z)+z\left(\partial^{2} f_{k}\right)(z)+(k+\delta+\xi)\left(\partial f_{k}\right)(z)\right)\right. \\
& \left.+(k+\delta+\xi) z\left(\partial f_{k}\right)(z)+(k+\delta+\xi)^{2} f_{k}(z)\right) X^{k+\delta}, \\
\left(e^{+} e^{-}\right) \cdot F(z, X)= & -\sum_{k=0}^{\infty} \partial\left(z^{2}\left(\partial f_{k}\right)(z)+2(k+\delta+\xi) z f_{k}(z)+f_{k-1}(z)\right) X^{k+\delta} \\
= & -\sum_{k=0}^{\infty}\left(2 z\left(\partial f_{k}\right)(z)+z^{2}\left(\partial^{2} f_{k}\right)(z)+2(k+\delta+\xi) f_{k}(z)\right. \\
& \left.+2(k+\delta+\xi) z\left(\partial f_{k}\right)(z)+\left(\partial f_{k-1}\right)(z)\right) X^{k+\delta}, \\
\left(e^{-} e^{+}\right) \cdot F(z, X)= & -\sum_{k=0}^{\infty}\left(z^{2}\left(\partial^{2} f_{k}\right)(z)+2(k+\delta+\xi) z\left(\partial f_{k}\right)(z)+\left(\partial f_{k-1}\right)(z)\right) X^{k+\delta}
\end{aligned}
$$

with $f_{-1}=0$. Thus we see that

$$
\mathcal{C} \cdot F(z, X)=\sum_{k=0}^{\infty}\left(2(k+\delta+\xi)^{2} f_{k}(z)-2(k+\delta+\xi) f_{k}(z)-2\left(\partial f_{k-1}\right)(z)\right) X^{k+\delta}
$$

which coincides with (27).

We now define the linear map $\Xi: \mathcal{F}[[X]]_{\delta} \rightarrow \mathcal{F}$ by

$$
\Xi(F(z, X))=(\Xi F)(z)=f_{0}(z)
$$

for $F(z, X)=\sum_{k=0}^{\infty} f_{k}(z) X^{k+\delta} \in \mathcal{F}[[X]]_{\delta}$. Then we see that the sequence

$$
0 \rightarrow \mathcal{F}[[X]]_{\delta+1} \stackrel{\iota}{\rightarrow} \mathcal{F}[[X]]_{\delta} \stackrel{\Xi}{\longrightarrow} \mathcal{F} \rightarrow 0
$$

is exact, where $\iota$ is the inclusion map. If $f: \mathcal{H} \rightarrow \mathbb{C}$ is a function on $\mathcal{H}$ and $\alpha$ is an integer, we set

$$
\left(\left.f\right|_{\alpha} \gamma\right)(z)=\mathfrak{J}(\gamma, z)^{-\alpha} f(\gamma z)
$$

for all $z \in \mathcal{H}$ and $\gamma \in \mathfrak{s l}(2, \mathbb{C})$, where $\mathfrak{J}(\gamma, z)$ is as in $(1)$. Then $\left.\right|_{\alpha}$ determines a right action of $\mathfrak{s l}(2, \mathbb{C})$ on $\mathcal{F}$, and from (3) and (29) we see easily that

$$
\Xi\left(\left.F\right|_{\lambda} ^{J} \gamma\right)=\left.(\Xi F)\right|_{2 \delta+\lambda} \gamma
$$


for $F(z, X) \in \mathcal{F}[[X]]_{\delta}$; hence $\Xi$ is $\operatorname{SL}(2, \mathbb{C})$-equivariant. We now assume that there is an $\mathrm{SL}(2, \mathbb{C})$-equivariant splitting $\eta: \mathcal{F} \rightarrow \mathcal{F}[[X]]_{\delta}$ of $\Xi$, meaning that $\eta$ a linear map satisfying

$$
\Xi(\eta f)=f, \quad \eta\left(\left.f\right|_{2 \delta+\lambda} \gamma\right)=\left.(\eta f)\right|_{\lambda} ^{J} \gamma
$$

for all $f \in \mathcal{F}$ and $\gamma \in \mathrm{SL}(2, \mathbb{C})$.

Proposition 4.2. If $\eta: \mathcal{F} \rightarrow \mathcal{F}[[X]]_{\delta}$ satisfies (30), then

$$
(\eta f)(z, X)=\sum_{k=0}^{\infty} \frac{f^{(k)}(z)}{(k+\delta+\xi) !(k+\delta+\xi-1) !} X^{k+\delta}
$$

for all $f \in \mathcal{F}$.

Proof. Given $f \in \mathcal{F}$, since $\eta$ satisfies (30), we have

$$
\eta(v \cdot f)=v \cdot(\eta f)
$$

for all $v \in \mathfrak{s l}(2, \mathbb{C})$ with respect to the action in (19). In particular, we obtain

$$
\eta(\mathcal{C} \cdot f)=\mathcal{C} \cdot(\eta f),
$$

where $\mathcal{C}$ is the Casimir operator in (26). Thus we see that

$$
\mathcal{C} \cdot f=(\Xi \circ \eta)(\mathcal{C} \cdot f)=\Xi(\mathcal{C} \cdot(\eta f)) .
$$

If $\eta f$ is given by

$$
(\eta f)(z, X)=\sum_{k=0}^{\infty} f_{k}(z) X^{k+\delta}
$$

with $f_{0}=f$, then from (27) we obtain

$$
\begin{aligned}
(\mathcal{C} \cdot(\eta f))(z, X) & =2(\delta+\xi)(\delta+\xi-1) f(z) X^{\delta} \\
& +2 \sum_{k=1}^{\infty}\left((k+\delta+\xi)(k+\delta+\xi-1) f_{k}(z)-\left(\partial f_{k-1}\right)(z)\right) X^{k+\delta} .
\end{aligned}
$$

Hence we have

$$
\mathcal{C} \cdot f=2(\delta+\xi)(\delta+\xi-1) f .
$$

From this, (32) and (33) we see that

$$
\begin{aligned}
0 & =(\eta(\mathcal{C} \cdot f))(z, X)-2(\delta+\xi)(\delta+\xi-1)(\eta f)(z) X^{\delta} \\
& =2 \sum_{k=1}^{\infty}\left((k+\delta+\xi)(k+\delta+\xi-1) f_{k}(z)-\left(\partial f_{k-1}\right)(z)\right) X^{k+\delta},
\end{aligned}
$$


which implies that

$$
f_{k}=\frac{\partial f_{k-1}}{(k+\delta+\xi)(k+\delta+\xi-1)}=\frac{f^{(k)}}{(k+\delta+\xi) !(k+\delta+\xi-1) !}
$$

for all $k \geq 1$. Hence the proposition follows by recursion.

Remark 4.3. Results similar to those of Proposition 4.1 and Proposition 4.2 were discussed by Cohen, Manin, and Zagier in [4, section 2] in terms of pseudodifferential operators.

\section{Lie algebras of Jacobi-like forms}

In this section we apply some of the results obtained in the previous sections to the case where the coefficients of the pseudodifferential operators and formal power series are holomorphic functions on the Poincaré upper half plane. We are interested in pseudodifferential operators and formal power series that are invariant under actions of discrete subgroups of $\operatorname{SL}(2, \mathbb{R})$, which leads us to the notion of automorphic pseudodifferential operators and Jacobi-like forms.

Let $\mathcal{H}$ be the Poincaré upper half plane on which $\mathrm{SL}(2, \mathbb{R})$ acts as usual by Möbius transformations, and let $R$ be the complex algebra of holomorphic function on $\mathcal{H}$. We denote by $R[[X]]$ the space of formal power series with coefficients in $R$ and by $\Psi \mathcal{D}(R)$ the space of pseudodifferential operators over $R$. Then the formulas (3) and (4) for $z \in \mathcal{H}$ and $\gamma \in \mathrm{SL}(2, \mathbb{R})$ determine right actions of $\mathrm{SL}(2, \mathbb{R})$ on $R[[X]]$ and $\Psi \mathcal{D}(R)$, respectively.

Definition 5.1. Let $\Gamma$ be a discrete subgroup of $\mathrm{SL}(2, \mathbb{R})$, and let $\lambda$ be an integer.

(i) A holomorphic function $f \in R$ is a modular form for $\Gamma$ of weight $\lambda$ if it satisfies

$$
\left(\left.f\right|_{\lambda} \gamma\right)(z)=f(z)
$$

for all $z \in \mathcal{H}$ and $\gamma \in \Gamma$, where $\left.f\right|_{\lambda} \gamma$ is as in (29). We denote by $\mathcal{M}_{\lambda}(\Gamma)$ the space of all modular forms for $\Gamma$ of weight $\lambda$.

(ii) Given an integer $\lambda$, a formal power series $\Phi(z, X) \in R[[X]]$ is a Jacobi-like form for $\Gamma$ of weight $\lambda$ if it satisfies

$$
\left(\left.\Phi\right|_{\lambda} ^{J} \gamma\right)(z, X)=\Phi(z, X)
$$

for all $z \in \mathcal{H}$ and $\gamma \in \Gamma$, where $\left.\Phi\right|_{\lambda} ^{J} \gamma$ is as in (3). The space of all Jacobi-like forms for $\Gamma$ of weight $\lambda$ is denoted by $\mathcal{J}_{\lambda}(\Gamma)$.

(iii) A pseudodifferential operator $\Psi(z) \Psi \mathcal{D}(R)$ is an automorphic pseudodifferential operator for $\Gamma$ if it satisfies

$$
(\Psi \circ \gamma)(z)=\Psi(z)
$$


for all $z \in \mathcal{H}$ and $\gamma \in \Gamma$, where $\Psi \circ \gamma$ is as in (4). We use $\Psi \mathcal{D}(R)^{\Gamma}$ to denote the subspace of $\Psi \mathcal{D}(R)$ consisting of all automorphic pseudodifferential operators for $\Gamma$.

If $\alpha, \beta \in \mathbb{Z}$ with $\alpha \geq 0$, we set

$$
R[[X]]_{\alpha}=X^{\alpha} R[[X]],
$$

and denote by $\Psi \mathcal{D}(R)_{\beta}$ the subspace of $\Psi \mathcal{D}(R)$ consisting of elements of the form

$$
\Psi(z)=\sum_{k=0}^{\infty} \psi_{k}(z) \partial^{\beta-k}
$$

with $\psi_{k} \in R$ for each $k \geq 0$. Then we see that the formulas (6) and (7) determine $\mathbb{C}$-linear isomorphisms

$$
\mathcal{L}_{\xi}^{\partial}: R[[X]]_{\delta} \rightarrow \Psi \mathcal{D}(R)_{-\delta-\xi}, \quad \mathcal{L}_{\xi}^{X}: \Psi \mathcal{D}(R)_{-\varepsilon} \rightarrow R[[X]]_{\varepsilon+\xi}
$$

for integers $\delta, \varepsilon>0$ and $\xi \geq 0$. Similarly, as in Theorem 2.1, the formula (13) determines the bilinear map

$$
[,]^{X}: R[[X]]_{\delta} \times R[[X]]_{\varepsilon} \rightarrow R[[X]]_{\delta+\varepsilon}
$$

satisfying

$$
\mathcal{L}_{2 \xi}^{\partial}\left([F(z, X), G(z, X)]^{X}\right)=\left[\mathcal{L}_{\xi}^{\partial}(F(z, X)), \mathcal{L}_{\xi}^{\partial}(G(z, X))\right]^{\partial}
$$

for $F(z, X) \in R[[X]]_{\delta}$ and $G(z, X) \in R[[X]]_{\varepsilon}$. Thus we see easily that $[,]^{X}$ is a Lie bracket on $R[[X]]$; hence $R[[X]]$ is a complex Lie algebra.

Given a discrete subgroup $\Gamma$ of $\operatorname{SL}(2, \mathbb{C})$, we set

$$
\mathcal{J}_{\lambda}(\Gamma)_{\alpha}=\mathcal{J}_{\lambda}(\Gamma) \cap R[[X]]_{\alpha}, \quad \Psi \mathcal{D}(R)_{\beta}^{\Gamma}=\Psi \mathcal{D}(R)^{\Gamma} \cap \Psi \mathcal{D}(R)_{\beta}
$$

for $\alpha, \beta \in \mathbb{Z}$ with $\alpha \geq 0$.

Proposition 5.2. The space $\mathcal{J}(\Gamma)$ of Jacobi-like forms for $\Gamma$ is a complex Lie algebra with respect to the bracket operation in (34) whose formula is given by (13).

Proof. As in Proposition 1.1, it can be shown that the two relations in (9) hold for $F(z, X) \in \mathcal{J}_{2 \xi}(\Gamma)_{\delta}, \Psi(z) \in \Psi \mathrm{DO}_{-\varepsilon}^{\Gamma}$ and $\gamma \in \mathrm{SL}(2, \mathbb{R})$. In particular, we have

$$
\left(\mathcal{L}_{\xi}^{\partial} F\right) \circ \gamma=\mathcal{L}_{\xi}^{\partial}\left(\left.F\right|_{2 \xi} ^{J} \gamma\right)=\mathcal{L}_{\xi}^{\partial} F,
$$

which shows that $\mathcal{L}_{\xi}^{\partial} F \in \Psi \mathrm{DO}_{-\delta-\xi}^{\Gamma}$. Similarly, the second relation in (9) shows that

$$
\left.\left(\mathcal{L}_{\xi}^{X} \Psi\right)\right|_{2 \xi} ^{J} \gamma=\mathcal{L}_{\xi}^{X}(\Psi \circ \gamma)=\mathcal{L}_{\xi}^{X} \Psi,
$$


and therefore $\mathcal{L}_{\xi}^{X} \Psi \in \mathcal{J}_{2 \xi}(\Gamma)_{\varepsilon-\xi}$. Thus we obtain the isomorphisms

$$
\mathcal{L}_{\xi}^{\partial}: \mathcal{J}_{2 \xi}(\Gamma)_{\delta} \rightarrow \Psi \mathrm{DO}_{-\delta-\xi}^{\Gamma}, \quad \mathcal{L}_{\xi}^{X}: \Psi \mathrm{DO}_{-\varepsilon}^{\Gamma} \rightarrow \mathcal{J}_{2 \xi}(\Gamma)_{\varepsilon-\xi}
$$

for each pair of nonnegative integers $\delta$ and $\varepsilon$. We now consider elements $F(z, X)$, $G(z, X) \in \mathcal{J}_{2 \xi}(\Gamma)_{\delta}$, so that

$$
\mathcal{L}_{\xi}^{\partial}(F(z, X)), \mathcal{L}_{\xi}^{\partial}(G(z, X)) \in \Psi \mathcal{D}(R)_{-\delta}^{\Gamma} .
$$

However, from (35) we see that

$$
[F(z, X), G(z, X)]^{X}=\left(\mathcal{L}_{2 \xi}^{\partial}\right)^{-1}\left[\mathcal{L}_{\xi}^{\partial}(F(z, X)), \mathcal{L}_{\xi}^{\partial}(G(z, X))\right]^{\partial} .
$$

Thus it follows that

$$
[F(z, X), G(z, X)]^{X} \in \mathcal{J}_{2 \xi}(\Gamma)_{2 \delta},
$$

which proves the proposition.

Given $\delta \geq 0$, as in (28) and (30), there is a short exact sequence

$$
0 \rightarrow R[[X]]_{\delta+1} \rightarrow R[[X]]_{\delta} \stackrel{\Xi}{\rightarrow} R \rightarrow 0,
$$

where the linear map $\Xi: R[[X]]_{\delta} \rightarrow R$ satisfies

$$
\Xi\left(\left.F\right|_{\lambda} ^{J} \gamma\right)=\left.(\Xi F)\right|_{2 \delta+\lambda} \gamma
$$

for all $\gamma \in \mathrm{SL}(2, \mathbb{R})$. By taking the $\Gamma$-invariant elements of the terms of the sequence (36) we obtain the short exact sequence

$$
0 \rightarrow \mathcal{J}_{\lambda}(\Gamma)_{\delta+1} \rightarrow \mathcal{J}_{\lambda}(\Gamma)_{\delta} \stackrel{\Xi}{\rightarrow} M_{2 \delta+\lambda} \rightarrow 0 .
$$

If $\eta: R \rightarrow R[[X]]_{\delta}$ is the linear map given by(31), then it satisfies

$$
\Xi(\eta f)=f, \quad \eta\left(\left.f\right|_{2 \delta+\lambda} \gamma\right)=\left.(\eta f)\right|_{\lambda} ^{J} \gamma
$$

for all $f \in R$ and $\gamma \in \operatorname{SL}(2, \mathbb{R})$. Hence, if $f \in M_{2 \delta+\lambda}$, we see that $\eta f$ is a Jacobilike form belonging to $\mathcal{J}_{\lambda}(\Gamma)_{\delta}$, so that the resulting map $\eta: M_{2 \delta+\lambda} \rightarrow \mathcal{J}_{\lambda}(\Gamma)_{\delta}$ is a splitting for the short exact sequence (36). Thus $\eta$ may be regraded as a lifting map from modular forms to Jacobi-like forms considered in [4]. On the other hand, considering the relations (9) over $R$, we obtain

$$
\left(\mathcal{L}_{\xi}^{\partial} F\right) \circ \gamma=\mathcal{L}_{\xi}^{\partial}\left(\left.F\right|_{2 \xi} ^{J} \gamma\right),\left.\quad\left(\mathcal{L}_{\xi}^{X} \Psi\right)\right|_{2 \xi} ^{J} \gamma=\mathcal{L}_{\xi}^{X}(\Psi \circ \gamma)
$$

for $F(z, X) \in R[[X]]_{\delta}, \Psi(z) \in \Psi \mathcal{D}(R)_{-\varepsilon}$ and $\gamma \in \mathrm{SL}(2, \mathbb{R})$. Thus we see that there is an isomorphism between $\mathcal{J}_{2 \xi}(\Gamma)_{\delta}$ and $\Psi \mathcal{D}(R)_{-\varepsilon}$; hence the lifting $\eta$ induces a lifting $\eta^{\partial}$ from modular forms to automorphic pseudodifferential operators.

Using the liftings of two modular forms via $\eta^{\partial}$ and the fact that the product of two $\Gamma$-automorphic pseudodifferential operators are $\Gamma$-automorphic, we obtain noncommutative products of modular forms known as Rankin-Cohen brackets (see $[4,15])$. The Rankin-Cohen brackets can also be interpreted in terms of classical transvectatnts (see, e.g., $[11,12]$ ). 


\section{References}

[1] M. Adler, On a trace functional for formal pseudo differential operators and the symplectic structure of the Korteweg-de Vries type equations, Invent. Math. 50 (1978/79), no. 3, 219-248.

[2] R. W. Carroll, Topics in soliton theory, North-Holland Mathematics Studies, vol. 167, NorthHolland Publishing Co., Amsterdam, 1991.

[3] I. Cherednik, Basic methods of soliton theory, Advanced Series in Mathematical Physics, vol. 25, World Scientific Publishing Co. Inc., River Edge, NJ, 1996.

[4] P. B. Cohen, Y. Manin, and D. Zagier, Automorphic pseudodifferential operators, Algebraic aspects of integrable systems, Progr. Nonlinear Differential Equations Appl., vol. 26, Birkhäuser Boston, Boston, MA, 1997, pp. 17-47.

[5] L. A. Dickey, Soliton equations and Hamiltonian systems, Advanced Series in Mathematical Physics, vol. 12, World Scientific Publishing Co. Inc., River Edge, NJ, 1991.

[6] M. Eichler and D. Zagier, The theory of Jacobi forms, Progress in Mathematics, vol. 55, Birkhäuser Boston Inc., Boston, MA, 1985.

[7] I. M. Gelfand and L. A. Dikij, Asymptotic behaviour of the resolvant of Sturm-Louville equations and the algebra of the Korteweg-de Vries equations, Russian Math. Surveys 30 (1975), 77-113.

[8] F. Gesztesy and H. Holden, Soliton equations and their algebro-geometric solutions, I: $(1+1)-$ dimensional continuous models, Cambridge Studies in Advanced Mathematics, vol. 79, Cambridge University Press, Cambridge, 2003.

[9] M. Kaneko and D. Zagier, A generalized Jacobi theta function and quasimodular forms, Progress in Mathematics, vol. 129, Birkhäuser Boston Inc., Boston, MA, 1995, pp. 165-172.

[10] B. A. Kupershmidt, KP or mKP: Noncommutative mathematics of Lagrangian, Hamiltonian, and integrable systems, Mathematical Surveys and Monographs, vol. 78, American Mathematical Society, Providence, RI, 2000.

[11] P. J. Olver, Classical invariant theory, London Mathematical Society Student Texts, vol. 44, Cambridge University Press, Cambridge, 1999.

[12] P. J. Olver and J. A. Sanders, Transvectants, modular forms, and the Heisenberg algebra, Adv. in Appl. Math. 25 (2000), no. 3, 252-283.

[13] G. Segal and G. Wilson, Loop groups and equations of KdV type, Inst. Hautes Études Sci. Publ. Math. (1985), no. 61, 5-65.

[14] G. Wilson, Commuting flows and conservation laws for Lax equations, Math. Proc. Cambridge Philos. Soc. 86 (1979), no. 1, 131-143.

[15] D. Zagier, Modular forms and differential operators, Proc. Indian Acad. Sci. Math. Sci. 104 (1994), no. 1, 57-75. K. G. Ramanathan memorial issue. 